\title{
Trends in MODIS geolocation error analysis
}

\author{
R. E. Wolfe ${ }^{\mathrm{a}}$, Masahiro Nishihama ${ }^{\mathrm{a}, \mathrm{b}}$ \\ ${ }^{a}$ Earth Sciences Directorate, NASA/GSFC, Greenbelt, MD 20771 \\ ${ }^{\mathrm{b}}$ Sigma Space Corporation, Lanham, MD, USA
}

\begin{abstract}
Data from the two MODIS instruments have been accurately geolocated (Earth located) to enable retrieval of global geophysical parameters. The authors describe the approach used to geolocate with sub-pixel accuracy over nine years of data from MODIS on NASA's EOS Terra spacecraft and seven years of data from MODIS on the Aqua spacecraft. The approach uses a geometric model of the MODIS instruments, accurate navigation (orbit and attitude) data and an accurate Earth terrain model to compute the location of each MODIS pixel. The error analysis approach automatically matches MODIS imagery with a global set of over 1,000 ground control points from the liner-resolution Landsat satellite to measure static biases and trends in the MODIS geometric model parameters. Both within orbit and yearly thermally induced cyclic variations in the pointing have been found as well as a general long-term trend.
\end{abstract}

Keywords: MODIS, EOS, Geolocation, Satellite navigation

\section{INTRODUCTION}

Since February 2000, the first Moderate Resolution Imaging Spectroradiometer (MODIS) instrument [1] has been acquiring images of the Earth in 36 spectral bands with nadir spatial resolutions of $0.25 \mathrm{~km}$ ( 2 bands), $0.5 \mathrm{~km}(5$ bands) and $1.0 \mathrm{~km}$ ( 29 bands). The center spectral wavelengths of these bands range from the visible $(0.41 \mu \mathrm{m})$ to the long-wave infrared $(14.2 \mu \mathrm{m})$. Two MODIS instruments are currently on-orbit. The first launched on NASA's Earth Observing System (EOS) Terra spacecraft in December 1999 and the second on NASA's EOS Aqua spacecraft in May 2002. Over nine years of data from MODIS/Terra and seven years of MODIS/Aqua data have been accurately geolocated to enable accurate retrieval of global geophysical parameters. In this paper, we discuss results from the geolocation error analysis approach that has allowed us to maintain and improve the geolocation accuracy for both instruments over their mission lifetimes.

The MODIS geolocation and error analysis approach is discussed in detail in [2] with the first on-orbit results presented in [3]. The approach uses a geometric model of the MODIS instruments, accurate navigation (orbit and attitude) data and an accurate Earth terrain model to compute the location of each MODIS pixel. The error analysis approach automatically matches MODIS imagery with a global set of 1,214 ground control points from the finerresolution Landsat satellite to measure static biases and trends in the MODIS geometric model parameters. Later geolocation results are also presented in [4], [5] and [6]. Both within-orbit and yearly thermally induced cyclic variations in the pointing have been found as well as a general long-term trend. Also, details of the control point matching algorithm are described in [6].

MODIS data has been reprocessed several times allowing for improvements in instrument characterization and algorithms to be incorporated in the standard MODIS products. The current reprocessing started in 2007 and is called Collection 5 (C5). The next reprocessing, Collection $6(\mathrm{C} 6)$, is expected to begin in early 2010 . For geolocation, each collection includes improvements in the geometric characterizations and minor algorithm improvements ([4], [5] and [6]). In addition, for C6, an improved Terrain correction algorithm [7] will be added. Starting with $\mathrm{C5}$, several improved ancillary data sets were incorporated: an improved control point library containing more than three times the number of control points with a better global distribution [6]; an improved digital terrain model based on the Shuttle Radar Terrain Mapping Mission (SRTM) data [8]; and an improved MODIS based Land/Water mask. In this paper, we discuss the MODIS/Terra and MODIS/Aqua geolocation results from the current $\mathrm{C} 5$ reprocessing and the results expected in $\mathrm{C} 6$. 


\section{MODIS/Terra Geolocation Results}

The MODIS/Terra C5 geolocation error (Table 1 and Figure 1) is small and meets the MODIS geolocation accuracy goal of $50 \mathrm{~m}(1 \mathrm{\sigma}), 20 \%$ of a MODIS $250 \mathrm{~m}$ band. Table 1 gives the C5 geolocation Root Mean Square Error (RMSE) in nadir equivalent units based on an average of 269 control point matchups per day in the track and scan (cross-track) direction. Shown in Figure 1 are the daily average control point residuals for each data-day since launch. Also shown are the global, northern hemisphere and southern hemisphere 16-day average control point residuals, along with the global 16-day standard deviation (gray bars). Overall, the long-term trend is close to zero, except for some periods, such as the period in late 2005 , when the overall trend in the scan direction did not follow the prediction. This was corrected

Table 1. MODIS control point residuals in nadir equivalent units for $\mathrm{C} 5$ and expected $\mathrm{C} 6$ results.

\begin{tabular}{|c|c|c|c|c|c|}
\hline \multirow{2}{*}{$\begin{array}{l}\text { Satel- } \\
\text { lite }\end{array}$} & \multirow{2}{*}{$\begin{array}{l}\text { Matches } \\
\text { per day }\end{array}$} & \multicolumn{2}{|c|}{ C5 Results (m) } & \multicolumn{2}{|c|}{$\begin{array}{c}\text { Expected } \mathrm{C} 6 \\
\text { Results }(\mathrm{m})\end{array}$} \\
\hline & & $\begin{array}{l}\text { Track } \\
\text { RMSE }\end{array}$ & $\begin{array}{l}\text { Scan } \\
\text { RMSE }\end{array}$ & $\begin{array}{l}\text { Track } \\
\text { RMSE }\end{array}$ & $\begin{array}{l}\text { Scan } \\
\text { RMSE }\end{array}$ \\
\hline Terra & 269 & 42 & 44 & 42 & 42 \\
\hline Aqua & 232 & 47 & 53 & 45 & 51 \\
\hline
\end{tabular}
in the next update that occurred in early 2006.

An improvement made for MODIS/Terra $\mathrm{C} 5$ was the introduction of a sun-angle correction to correct for withinorbit variation seen in the ground control point residuals. Because of this correction, as seen in Figure 1 , the Northern and Southern hemisphere trends are not significantly different from the global trend.

Based on the $\mathrm{C} 5$ results, two improvements will be made in $\mathrm{C} 6$. First, a retrospective long-term trend analysis will be applied to remove any biases or extrapolation errors. Second, an improved sun-angle correction will be applied to correct for residual within-orbit variation. Table 1 and Figure 2 show the expected results from these changes. The overall C6 results are expected to improve in the scan direction, reducing the RMSE by $2 \mathrm{~m}$. This is primarily a result of the improved retrospective fit in 2005. The long-term trend includes a yearly cyclic term with a peak-topeak amplitude of $26 \mathrm{~m}$ in the track and $10 \mathrm{~m}$ in the scan direction. This amplitude will be $59 \%$ and $29 \%$ higher in
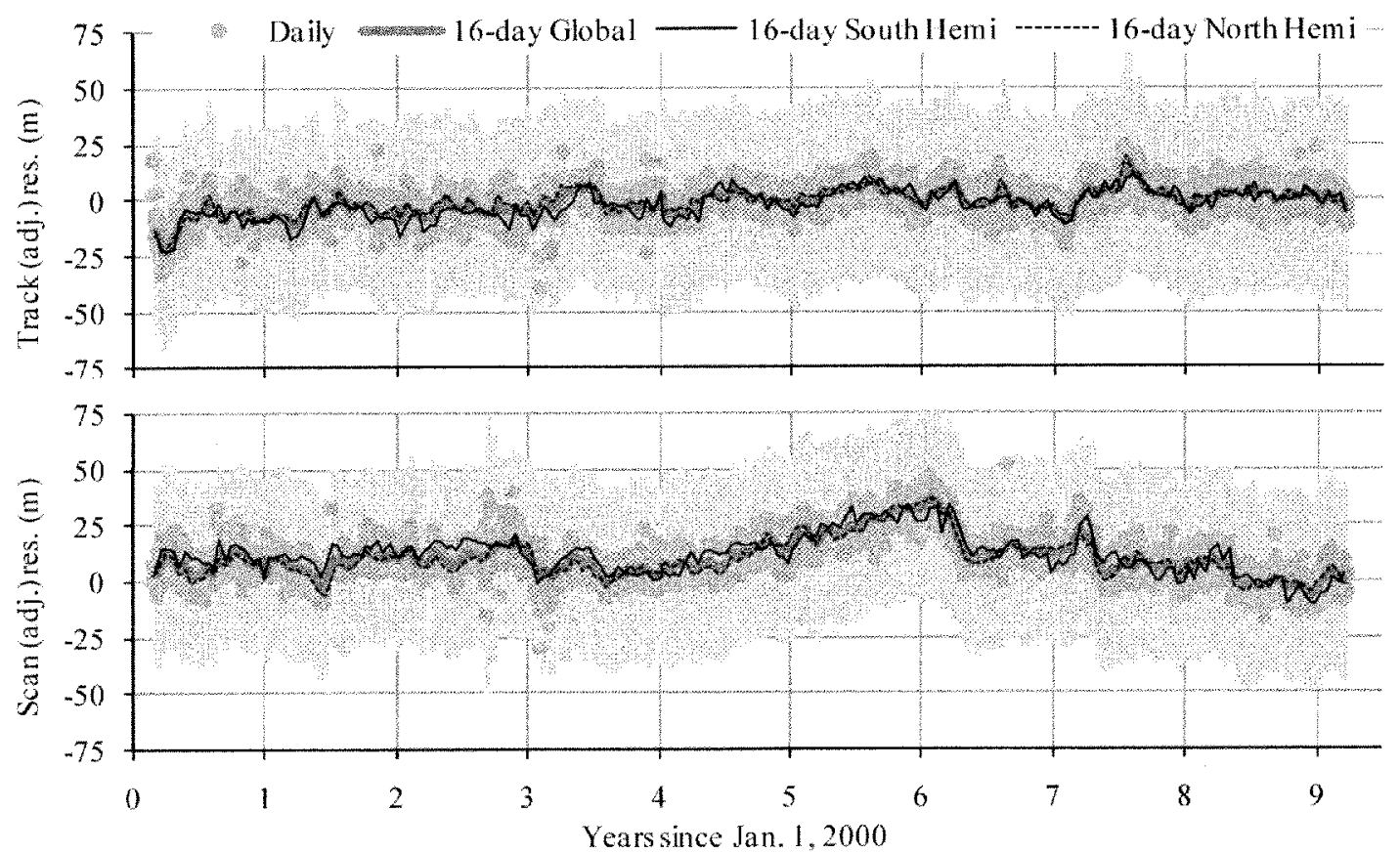

Figure 1. MODIS/Terra C5 control point residuals in the track (top) and scan (bottom) directions. 

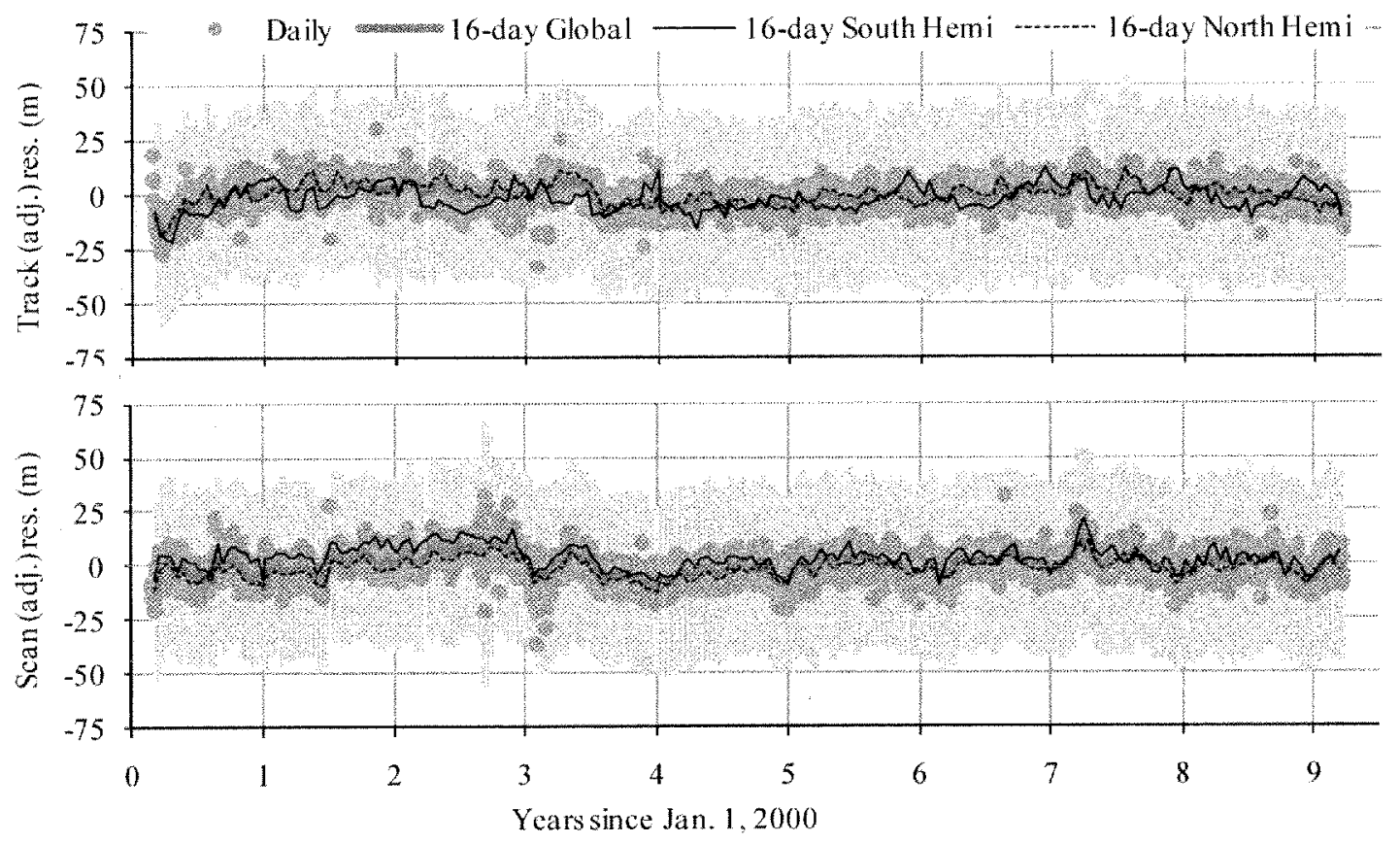

Figure 2. Expected MODIS/Terra C6 control point residuals in the track (top) and scan (bottom)

the track and scan directions, respectively, than $\mathrm{C} 5$.

The improved sun-angle fit is shown in Figure 3 (black lines). The control point residuals in nadir equivalent units are binned in $5^{\circ}$ increments (gray filled circles) and displayed with each bin's standard deviation (gray bars). The polynomial fits are used for the sun-angle range from $0^{\circ}$ to $120^{\circ}$ and then a linear correction for remainder of the orbit $\left(120^{\circ}\right.$ to $\left.360^{\circ}\right)$. The fit has a peak-to-peak amplitude of $40 \mathrm{~m}$ in the track and $41 \mathrm{~m}$ in the scan direction. This amplitude is $43 \%$ and $32 \%$ smaller in the track and scan directions, respectively, and so compensates for an overcorrection that occurred in C5.
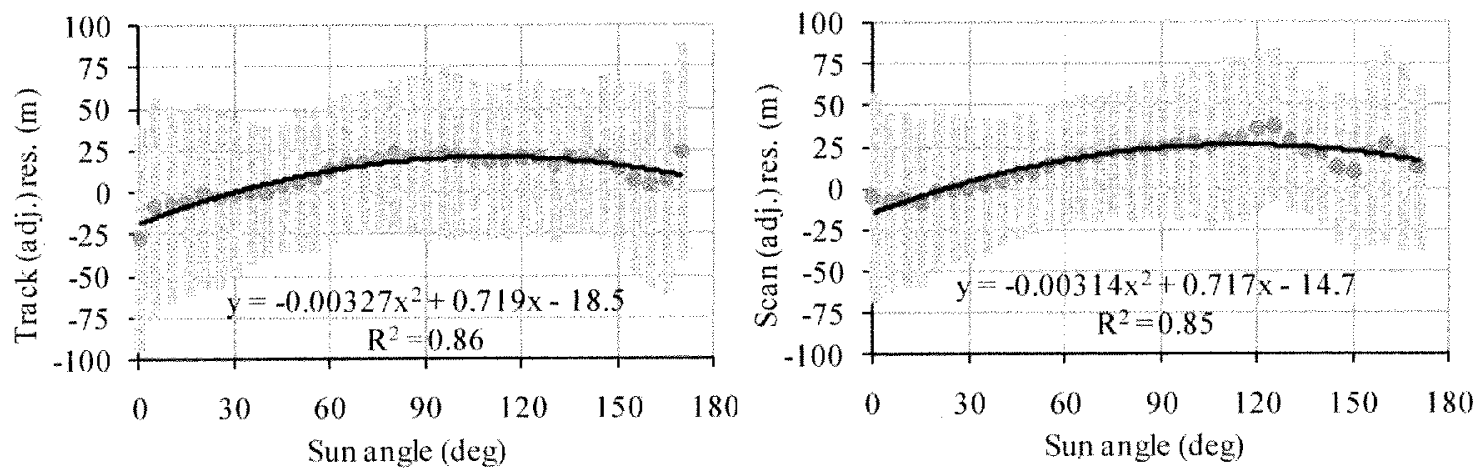

Figure 3. MODIS/Terra sun-angle fit in the track (left) and scan (right) directions.

As part of this error analysis, a study was done to determine whether instrument temperature could be used to correct for with-orbit pointing errors since temperature changes are believed to be the primary cause of the instrument and spacecraft pointing changes within an orbit. After removing the long-term trend from both the residuals and the temperature data from six instrument locations, the temperature that had the highest correlation with the control point residuals was the scan mitror temperature (TP SA RCT1_MIR thermistor). The relationship 
between this temperature and the pointing error was $-5.3 \mathrm{~m}$ and $-5.6 \mathrm{~m}$ per $1^{\circ} \mathrm{C}$ in the track and scan directions, respectively. When this temperature fit was used to remove this within-orbit variation, the overall results were not quite as good as the sun-angle fit. Based on this and because of the additional work needed to continuously check the quality of the temperature values, the sun-angle fit is the approach that will be used for C6.

\section{MODIS/Aqua Geolocation Results}

The MODIS/Aqua C5 geolocation error (Table 1 and Figure 4) is small and meets the MODIS geolocation accuracy goal in the scan direction and almost meets the goal in the track direction. Table 1 gives the C5 geolocation Root Mean Square Error (RMSE) in nadir equivalent units based on an average of 232 control point matchups per day in the track and scan (cross-track) direction. Overall, the long-term trend is close to zero, except for some periods, such as the period in 2007 and 2008, when the overall trend in the scan direction did not follow the prediction. This was corrected in the next update that occurred in early 2006. For Aqua, there is a significant with-orbit variation that causes the northern and southern hemisphere residuals to vary with respect to one another. In addition, there is a small global yearly cyclic term. Neither of these corrections was used in C5 for MODIS/Aqua.
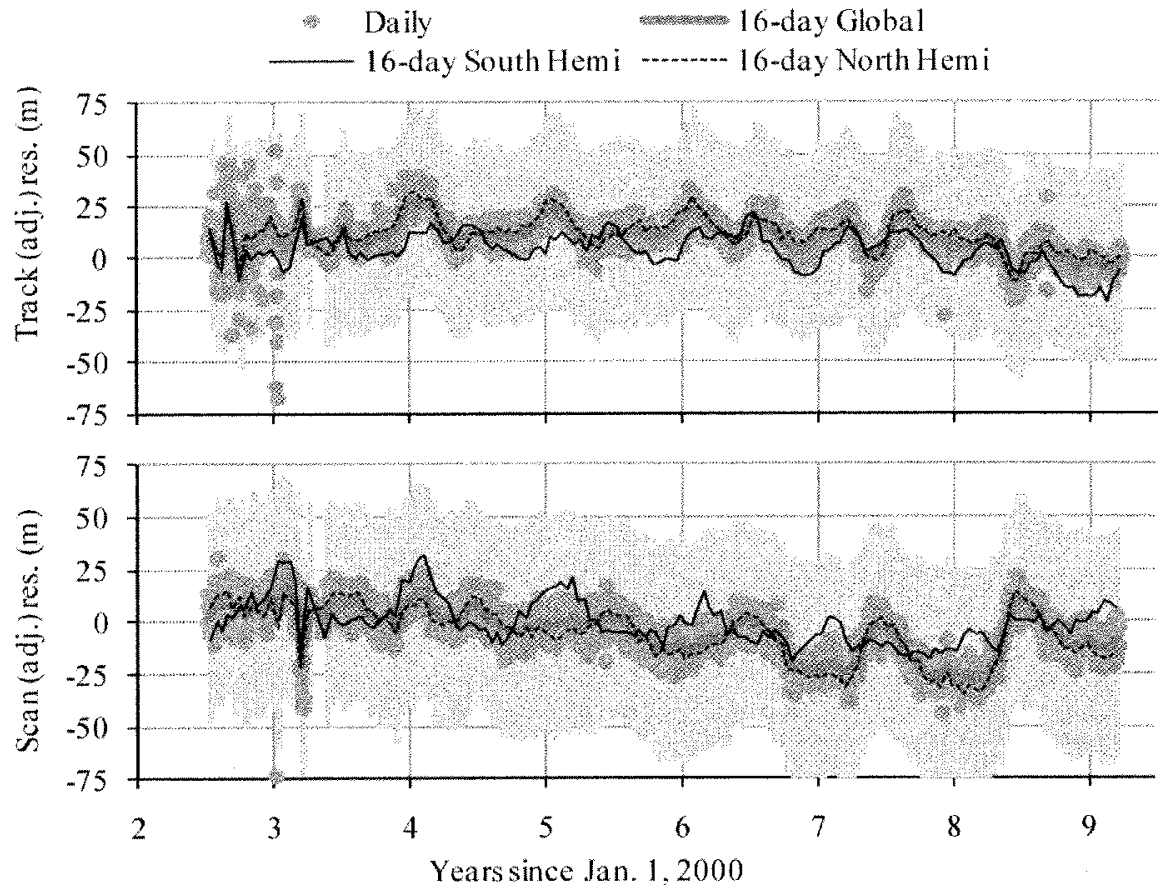

Figure 4. MODIS/Aqua C5 control point residuals in the track (top) and scan (bottom) directions.

Based on the C5 results, three improvements will be made in C6. First, a retrospective long-term trend analysis will be applied to remove any biases or extrapolation errors. Second, a yearly cyclic fit will be used to remove the global yearly variation. The third change will be to implement a sun-angle correction to correct for the within-orbit variation. Table 1 and Figure 5 show the expected results from these changes. The overall $C 6$ results are expected to improve in both the track and scan directions, reducing the RMSE by $2 \mathrm{~m}$ for each. The new long-term trend yearly cyclic terms will have small, peak-to-peak amplitudes of $1 \mathrm{~m}$ in the track and $5 \mathrm{~m}$ in the scan direction. As seen in Figure 5, the retrospective fit of the long-term trend that includes the cyclic term bring the global residuals close to zero in both the track and scan directions. In the track direction, almost all of the within-orbit variation is removed by the $26 \mathrm{~m}$ peak-to-peak amplitude sun-angle correction (Figure 6). There appears to be a small, twocycle per year residual error remaining in the track direction. In the scan direction, the within-orbit variation is reduced by the $62 \mathrm{~m}$ peak-to-peak sun-angle correction. However, there remains a small north/south difference that 
appears early in each year starting with 2004. Some additional adjustments will also be made in the early postlaunch period to correct for the initial on-orbit bias.

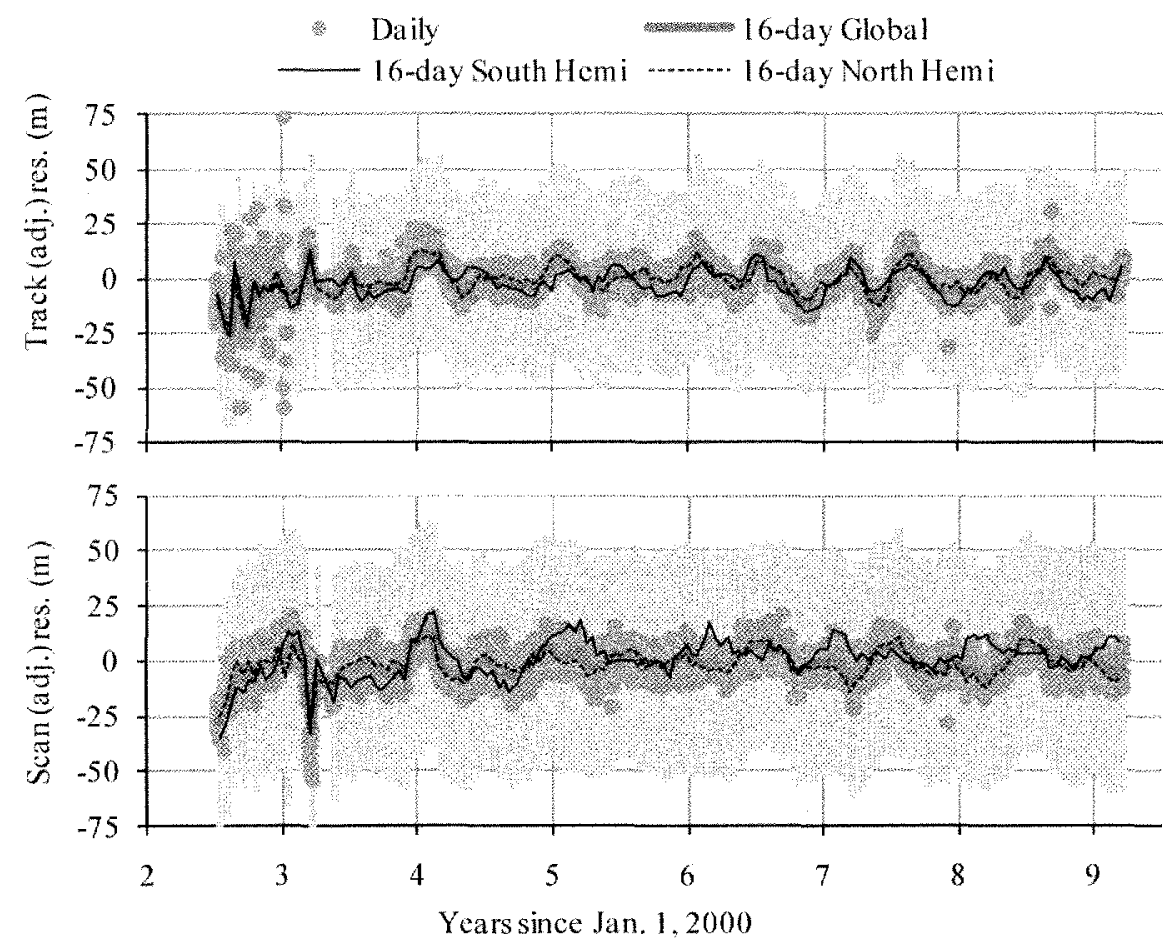

Figure 5. Expected MODIS/Aqua C6 control point residuals in the track (top) and scan (bottom) directions.

The first MODIS/Aqua sun-angle fit is shown in Figure 6. The polynomial fits are used for the sun-angle range from $0^{\circ}$ to $140^{\circ}$ and then a linear correction for remainder of the orbit $\left(140^{\circ}\right.$ to $\left.360^{\circ}\right)$. The MODIS/Aqua scan direction sun-angle fit is similar in sign and has a $51 \%$ larger amplitude than the MODIS/Terra scan fit. The MODIS/Aqua track direction sun-angle fit, however, has the opposite sign and an amplitude that is $35 \%$ smaller than the MODIS/Terra track fit.
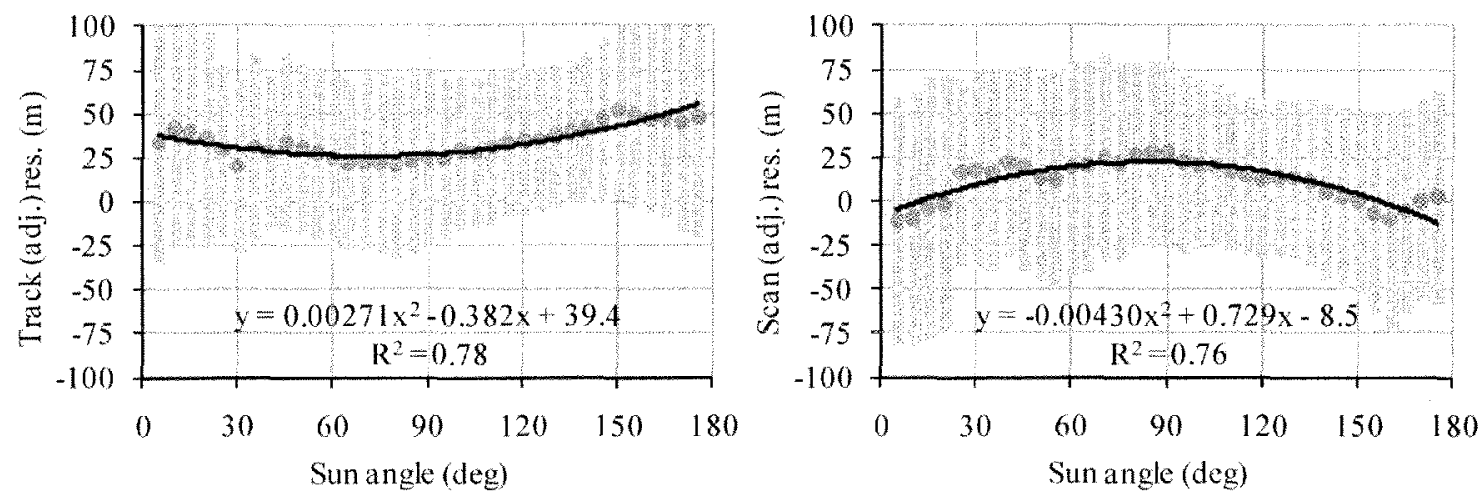

Figure 6. MODIS/Aqua sun-angle fit in the track (left) and scan (right) directions.

As part of the MODIS/Aqua C5 error analysis, a study like the study for MODIS/Terra was done to determine whether instrument temperature could be used to correct for within-orbit pointing errors. After removing the long- 
term trend from both the residuals and the temperature data from six instrument locations, there was no temperature that had a good correlation with the control point residuals. So, for MODIS/Aqua, no correction based on temperature was possible.

\section{Other Collection 6 Changes}

Several additional changes will be made for geolocation in C6. First, minor improvements in the overall geolocation accuracy will be obtained by making small adjustments in several instrument parameters (i.e. yaw bias, pitch bias, scan rate). Second, as mentioned above, an improved terrain correction algorithm [7] will be implemented that improves $1 \mathrm{~km}$ geolocation accuracy in areas with rough terrain. This change will be coupled with a higher quality terrain model and an improved land/water mask, both with $0.5 \mathrm{~km}$ resolution. Finally, in addition to the $1 \mathrm{~km}$ geolocation, geolocation will be computed at $0.5 \mathrm{~km}$ and stored as offsets from the $1 \mathrm{~km}$ geolocation values.

\section{Other Geolocation Considerations}

Both the Terra and Aqua spacecraft perform monthly attitude roll maneuvers for calibration purposes. Additionally, orbit maneuvers, drag makeup and inclination adjustment, are performed several times a year. For a short time before a maneuver and for up to 1.5 hours after a maneuver, the satellite orbit and attitude data are not as accurate, so the geolocation has larger errors than mentioned above. Because of this, these maneuver periods are excluded when the standard MODIS products are produced in C5. A different approach is being considered for C6 that would allow more data near maneuvers to be used.

For near-real-time users of MODIS/Aqua data, the predicted orbit data provided in the direct broadcast data stream is not as accurate as the definitive orbit data that is available 12 to 24 hours later. This is not an issue for MODIS/Terra because the direct broadcast data stream contains definitive orbit data.

\section{Summary and Conclusion}

The error analysis approach described above is being used to accurately geolocate data from both MODIS instruments. The current C5 geolocation results were discussed along with the geolocation improvements that are expected in the next MODIS reprocessing (C6) set to begin in early 2010. Overall, the accuracy of the MODIS geolocation data meets the tough accuracy goal set by the MODIS Science Team. The consistent sub-pixel geolocation accuracy enables the accurate retrieval of geophysical parameters globally. Lessons learned from this error analysis approach is being used for the Visible Infrared Imager Radiometer Suite (VIIRS) instruments aboard the National Polar-orbiting Operational Environmental Satellite System (NPOESS) Preparatory Project (NPP) and will be used for future operational NPOESS missions.

\section{Acknowledgement}

The authors are grateful for the support of the MODIS science team, the MODIS calibration support team, the MODAPS production team and the MODIS science data support team, in particular James Kuyper. This work was performed under the direction of the MODIS Science Team in the Terrestrial Information Systems Branch (Code $614.5)$ at NASA GSFC.

\section{References}

[1] Salomonson, V. V., Barnes, W. L., Maymon, P. W., Montgomery, H. E. and Ostrow, H., "MODIS: Advanced Facility Instrument for Studies of the Earth as a System," IEEE Trans. Geosci. Remote Sensing, 27, pp. 145-153 (1989).

[2] Nishihama, M., Wolfe, R. E., Solomon, D., Patt, F. S., Blanchette, J., Fleig A. J. and Masuoka, E., "MODIS Level IA Earth Location Algorithm Theoretical Basis Document Version 3.0, SDST-092," Laboratory for Terrestrial Physics, NASA Goddard Space Flight Center, Greenbelt, Maryland (1997). 
[3] Wolfe, R. E., Nishihama, M., Fleig, A. J., Kuyper, J. A., Roy, D. P., Storey, J. C. and Patt, F. S., "Achieving sub-pixel geolocation accuracy in support of MODIS land science," Remote Sensing of Environment, 83, pp. $31-49$ (2002).

14] Salomonson, V. V. and Wolfe, R. E., "MODIS Geolocation Approach, Results and the Future", IEEE Workshop on Advances in Techniques for Analysis of Remotely Sensed Data, NASA GSFC, Geeenbelt, MD, 424-427, Oct. 27-28 (2003).

[5] Wolfe, R. E., Chapter 4: MODIS Geolocation, [Earth Science Satellite Remote Sensing, Vol. 1], Geosciences of Springer-Verlag and Tsinghua University Press, pp. 50-71 (2006).

[6] Wolfe, R. E. and Nishihama, M., Book chapter: Accurate MODIS global geolocation through automated ground control image matching, [Image Registration for Remote Sensing], pending publication.

[7] Wolfe, R. E., Nishihama, M. and Kuyper, J. R., "Improving satellite moderate resolution instrument geolocation accuracy in rough terrain," IGARSS 2006: IEEE International Geosci. and Remote Sens. Sympositum, 11231125 , July (2006).

[8] Hilland, J. E., Stuhr, F. V., Freedman, A., Imel, D., Shen, Y., Jordan, R. and Caro, E., "Future NASA Spaceborne SAR Missions," IEEE Areospace and Electronic Systems Magazine, November, 9-16 (1998). 